\title{
Nota farmacológica: Rol de la glucosamina en el tratamiento de la osteoartritis
}

Role of glucosamine in the treatment of osteoarthritis

Yamila De Vas* y Victoria Wurcel*

\begin{abstract}
Resumen
La osteoartritis es una enfermedad degenerativa del cartílago que produce disminución del espacio articular y cambios en el hueso subyacente. Se postula que la administración de glucosamina exógena estimularía la síntesis de matriz cartilaginosa y protegería el hueso. A pesar de esto, no hay evidencia sólida para sostener el uso de glucosamina en la osteoartritis leve.

Los estudios mayores y de buena calidad metodológica indican que el beneficio sintomático es mínimo o inexistente; mientras que el efecto sobre la modificación en el espacio articular radiológico, valorado en pocos trabajos que adolecen de fallas metodológicas, es escaso y de significado incierto. En los casos de síntomas moderados o severos se observó una tendencia beneficiosa en la disminución de los síntomas con la terapia combinada de glucosamina más condroitín sulfato, aunque son resultados que deben confirmarse con estudios apropiados. La glucosamina es segura pero no es posible recomendarla como primera línea de tratamiento.

\section{Abstract}

Osteoarthritis is a degenerative disease of the cartilage that produces decreased joint space and changes in the underlying bone. It's hypothetized that administration of exogenous glucosamine stimulate the synthesis of cartilage matrix and protects the bone. Despite this, there is no evidence to support the use of glucosamine in mild osteoarthritis. The largest studies of good methodological quality indicate that symptomatic benefit is minimal or nonexistent, while the effect on the change in radiological joint space, valued in a few studies that suffer from methodological flaws, it is poor and of uncertain significance. In cases of moderate to severe symptoms the combination therapy of glucosamine plus chondroitin sulfate showed a beneficial trend, although results should be confirmed with appropriate studies. Glucosamine is safe but can not be recommended as first line of treatment.
\end{abstract}

Palabras clave: osteoartritis, glucosamina, dolor, pronóstico. Key words: osteoarthritis, glucosamine, pain, prognosis.
De Vas Y y Wurcel V. Rol de la glucosamina en el tratamiento de la osteoartritis. Evid. Act. Pract.Ambul. Vol 12(1). 28-30. Ene-Mar 2009.

\section{Introducción}

La osteoartritis es una enfermedad degenerativa del cartílago que produce disminución del espacio articular y cambios en el hueso subyacente. Afecta a más de 20 millones de pacientes en Estados unidos y su incidencia aumenta con la edad. La enfermedad es más frecuente en mujeres y causa importante morbilidad por dolor y pérdida de la función articular.

El tratamiento tiene como objetivo mejorar el dolor, intentar corregir los alineamientos articulares anómalos e identificar y corregir las manifestaciones de la inestabilidad articular. Actualmente se recomienda el mantenimiento del peso ideal, el apoyo con un bastón en forma contralateral al dolor, la fisioterapia, la terapia ocupacional y los analgésicos.

Sin embargo, estas medidas sintomáticas no revierten el proceso degenerativo, y el tratamiento con antinflamatorios no esteroides (AINE) se asocia a morbi-mortalidad por compromiso gastrointestinal y renal.

En este contexto es interesante la búsqueda de tratamientos farmacológicos más seguros para aliviar el dolor y permitir una mejor función de la articulación afectada, como es el caso de la glucosamina. Sin embargo la evidencia disponible sobre su eficacia y seguridad es controvertida.

\section{¿Cuál es el mecanismo de acción propuesto para la glu- cosamina?}

La administración de glucosamina exógena estimularía la síntesis de matriz cartilaginosa de proteoglucanos, que actúa como lubricante y protege al cartílago, confiriéndole resistencia contra la tensión mecánica y brindando protección inespecífica contra el daño químico. Su administración como sal sulfato proporcionaría al cartílago articular la hexosamina precursora y el anión sulfato necesarios para la síntesis de glucosaminoglucanos.
¿Cuál es la evidencia a corto plazo (ocho a 12 semanas) sobre la eficacia y la seguridad del tratamiento con glucosamina para la mejoría del dolor y la funcionalidad articular?

Una revisión sistemática de la Colaboración Cochrane ${ }^{1}$ examinó los estudios realizados hasta el año 2005 sobre glucosamina en osteoartritis. Fueron incluidos 20 estudios que habían evaluado este tratamiento en más de 2500 personas con osteoartritis de rodilla o cadera. La duración de la mayoría de los estudios fue de dos a tres meses, y los investigadores compararon glucosamina contra placebo o AINE.

\section{Resultados}

Dolor: los estudios de alta calidad mostraron que el dolor mejoró en la misma magnitud con glucosamina y con placebo. Luego de examinar todos los estudios (incluídos los de baja calidad metodológica e incluyendo también los más antiguos) pudo evidenciarse una mejoría en el dolor de 13 puntos más (sobre una escala de 0 a 100) en los pacientes que recibieron glucosamina que en quienes recibieron placebo o AINE (un $28 \%$ de cambio en relación al puntaje basal en la mejoría del dolor, con una diferencia media estandarizada de -0,61, (IC95\% -0,95 a -0,28).

Funcionalidad: los estudios de alta calidad mostraron que la glucosamina mejoraba la funcionalidad más que el placebo o los AINE, cuando se la medía a través de una escala $21 \%$ de mejoría de la función en relación al basal utilizando el índice de Lequesne, con una diferencia media estandarizada de -0.51; (IC95\% -0.96 a -0.05) pero no había diferencias cuando se la medía por otra escala (WOMAC).

El resultado fue similar cuando se analizaron todos los estudios (incluidos los de baja calidad y los antiguos) o sólo los de alta calidad metodológica, y respecto de la seguridad de las drogas, no hubo diferencias en relación al número de personas que sufrieron eventos adversos entre las diferentes ramas. Los efectos secundarios incluyeron principalmente trastornos estomacales y dolor articular. 


\section{Conclusiones}

La mejoría del dolor no mostró asociación al consumo de placebo, AINE o de glucosamina durante dos a tres meses. Dependiendo de la escala utilizada para medir la funcionalidad (capacidad física) ésta puede no mejorar en absoluto o mejorar en forma leve con el uso de glucosamina. La glucosamina parece ser segura.

\section{¿Cuál es la evidencia a mediano plazo (24 semanas) sobre la eficacia y la seguridad del tratamiento con glucosamina para la mejoría del dolor articular?}

El GAIT ${ }^{2}$ es un estudio multicéntrico, aleatorizado, doble ciego, controlado con placebo y celecobix, publicado en 2006 (por lo que no fue incluído en la revisión sistemática de la Colaboración Cochrane que hemos resumido previamente) que evaluó la eficacia de la glucosamina, el condroitín sulfato (otro precursor de la formación de la matríz cartilaginosa) o la combinación de ambos para el tratamiento del dolor provocado por la osteoartritis de rodilla.

\section{Métodos}

Fueron seleccionaron 1.583 pacientes con osteoartritis de rodilla sintomática y fueron asignados aleatoriamente a recibir una de las siguientes cinco intervenciones durante 24 semanas: 1) $1.500 \mathrm{mg} /$ día de glucosamina; 2) $1.200 \mathrm{mg}$ al día de condroitín sulfato; 3) $200 \mathrm{mg}$ al día de Celecoxib; 4) 1.500 mg día de glucosamina más $1200 \mathrm{mg}$ de condroitín sulfato; 5) placebo.

Como medicación de rescate se empleó paracetamol hasta una dosis de cuatro gramos al día.

Los pacientes tenían 40 años o más y presentaban dolor de rodilla con diagnóstico radiológico de osteoartritis. El puntaje de los síntomas en la escala WOMAC (cuestionario validado que evalúa dolor, rigidez y limitación funcional) fue de 125 a 400 puntos.

Según la intensidad del dolor, fueron subdivididos en dos grupos: intensidad leve $(n=1.229)$ e intensidad moderada a severa $(n=354)$. La variable principal del estudio fue la respuesta al tratamiento, definida previamente como el descenso de un $20 \%$ en la puntuación basal de la escala de dolor.

\section{Resultados}

El promedio de edad fue de 59 años, con un $64 \%$ de la muestra de sexo femenino. Los resultados del uso aislado de glucosamina y condroitín sulfato no fueron significativamente superiores al los de placebo en la reducción de la intensidad del dolor de rodilla.

La tasa de respuesta del tratamiento combinado fue un $6,5 \%$ superior a la del placebo $(p=0,009)$; mientras que la tasa de respuesta a celecoxib en el grupo control fue un $10 \%$ superior a la de placebo $(p=0,008)$ pero no se logró demostrar un descenso del $20 \%$ en la intensidad del dolor -que había sido el resultado planificado por los investigadores en el diseño del ensayo clínico- por lo que se concluyó que ninguno de ellos por separado ni la combinación de glucosamina más condroitín sulfato fueron más eficaces que el placebo.

En el subgrupo de pacientes con dolor de intensidad moderada a severa, la respuesta del tratamiento combinado fue significativamente mayor que la respuesta a placebo $(72,25 \%$ frente a $54,3 \% ; p=0,002)$.

\section{Discusión}

Este estudio tiene varias limitaciones. Por un lado, la elevada tasa de respuesta al placebo y la baja intensidad del dolor presentada por los pacientes puede haber disminuido la capacidad del ensayo para valorar la eficacia del tratamiento. Por otro lado, los efectos observados en el subgrupo de pacientes con dolor de moderado a severo no son completamente concluyentes debido al reducido número de pacientes en este subgrupo.

\section{Conclusiones}

Ni la glucosamina, ni el condroitín Sulfato, o la terapia de combinación confiere beneficio en términos de reducción significativa del dolor en pacientes con osteoartritis de rodilla leve. Se observó beneficio en la rama de la terapia combinada, en el subgrupo con síntomas moderados o graves, pero los resultados no son concluyentes debido a que es un análisis hecho a posteriori en un subgrupo pequeño de pacientes. Es necesaria la realización de un estudio diseñado específicamente para este subgrupo de pacientes antes de poder extraer conclusiones válidas.

\section{¿Cuál es la evidencia a largo plazo (tres años) sobre la efi- cacia y la seguridad del tratamiento con glucosamina para la mejoría del dolor y la estrechez articular?}

Otro estudio publicado en Lancet $2001^{3,4}$ e incluído en la revisión Cochrane antes mencionada, tuvo como objetivo evaluar los efectos a largo plazo de la administración de sulfato de glucosamina sobre la progresión de los síntomas y las alteraciones estructurales de la artrosis a través de un ensayo clínico, doble ciego, aleatorizado y controlado con placebo en Bélgica. Nos detendremos en él porque tuvo un seguimiento prolongado de los pacientes.

\section{Métodos}

Fueron incluidos 212 pacientes mayores de 50 años, con osteoartritis primaria de la rodilla del compartimiento femorotibial medial, documentada de acuerdo a criterios clínicos y radiológicos. Los pacientes fueron aleatorizados a dos grupos para recibir $1500 \mathrm{mg} /$ día de glucosamina o placebo, en una toma diaria y durante tres años.

\section{Medición de resultados principales}

El ancho del espacio articular fue evaluado mediante radiografías al inicio, al año y a los tres años de comenzado el protocolo. Los síntomas fueron evaluados mediante el índice WOMAC, valorándose el cambio porcentual del mismo a los tres años.

\section{Resultados principales}

Los pacientes tratados con glucosamina no presentaron estrechamiento significativo del espacio articular, mientras que en el grupo asignado a placebo se observó una mayor tendencia a desarrollar estrechamiento significativo (definido como un espacio articular menor a $0,5 \mathrm{~mm}$ ) a los 3 años.

Se observó mejoría de los síntomas clínicos en los pacientes tratados con Glucosamina, con respecto a los valores basales y en comparación con el grupo placebo, e independientemente de los cambios estructurales.

\section{Discusión}

Los cambios positivos en la valoración clínica en el grupo glucosamina fueron significativos en las sub-escalas de dolor y de función física, pero no en la de rigidez. Por el contrario, los pacientes del grupo placebo presentaron empeoramiento clínico. Los cambios en la estrechez articular observados habitualmente durante la progresión de la osteoartritis son de un rango muy amplio, siendo escasa su correlación con la clínica. Por lo 
tanto, la utilidad de este parámetro para la evaluación del tratamiento todavía no es clara. Además la técnica radiológica utilizada en este estudio ha sido cuestionada, por lo que produce incertidumbre sobre los resultados.

Aunque en este trabajo los beneficios del tratamiento se prolongan hasta tres años, todavía queda por determinar si estos efectos pueden afectar el pronóstico de la enfermedad. Sería de utilidad valorar el retraso en la aparición de invalidez o en la indicación de tratamiento quirúrgico.

\section{Conclusiones}

No hay evidencia sólida para respaldar el uso de glucosamina en la osteoartritis leve. Los estudios mayores y de buena calidad metodológica indican que el beneficio sintomático es mínimo o inexistente; mientras que el efecto sobre la modificación en el espacio articular radiológico, valorado en pocos trabajos que adolecen de fallas metodológicas, es escaso y de significado incierto.
En los casos de síntomas moderados o severos se observó una tendencia beneficiosa en la disminución de los síntomas con la terapia combinada de glucosamina más condroitín sulfato, aunque son resultados que deben confirmarse con estudios apropiados.

Datos de seguimientos a tres años indican que la glucosamina parece ser una medicación bien tolerada y segura.

A la hora de decidir la indicación de este tipo de tratamiento, además de tener en cuenta su eficacia y seguridad, es importante considerar su costo. Por ejemplo en Argentina, un tratamiento mensual con una dosis diaria de $1500 \mathrm{mg}$ de sulfato de glucosamina sóla o en combinación con condroitín sulfato cuesta entre u\$s 18 a 35 , monto elevado para un paciente añoso que muy probablemente deberá priorizar el destino de su dinero al tratamiento de otras patologías crónicas tan prevalentes durante la vejez. Por lo tanto y de acuerdo a la evidencia con que contamos hasta el momento, no nos parece razonable recomendar el uso de glucosamina como primera línea de tratamiento para los pacientes con osteoartritis.

\section{Referencias}

1. Towheed T y col. Glucosamine therapy for treating osteoarthritis (Cochrane Review). In: The Cochrane Library, Issue 4, 2008. Oxford: Update Software.

2. Clegg D y col. Glucosamine, chondroitin sulfate, and the two in combination for painful knee osteoarthritis. N Engl J Med. 2006; 354:795-808.

3. Reginster $\mathrm{J}$ y col. Long-term effects of glucosamine sulphate on osteoarthritis progresion: a randomized, placebo-controlled clinical trial. Lancet 2001, 357:251-56.

4. Villafañe, H. Efectos del Sulfato de glucosamina sobre la progresión de la artrosis. Evid. Act. Práct. Ambul. Vol. 5 Nro. Marzo-Abril 2002.Comentado de Reginster J y col.

Long-term effects of glucosamine sulphate on osteoarthritis progresion: a randomized, placebo-controlled clinical trial. Lancet 2001, 357:251-56. 